\title{
Scaling and width distributions of parity-conserving interfaces
}

\author{
M. Arlego and M. D. Grynberg \\ Departamento de Física, Universidad Nacional de La Plata, 1900 La Plata, Argentina
}

(Received 20 September 2013; published 27 November 2013)

\begin{abstract}
We present an alternative finite-size approach to a set of parity-conserving interfaces involving attachment, dissociation, and detachment of extended objects in $1+1$ dimensions. With the aid of a nonlocal construct introduced by Barma and Dhar in related systems [Phys. Rev. Lett. 73, 2135 (1994)], we circumvent the subdiffusive dynamics and examine close-to-equilibrium aspects of these interfaces by assembling states of much smaller, numerically accessible scales. As a result, roughening exponents, height correlations, and width distributions exhibiting universal scaling functions are evaluated for interfaces virtually grown out of dimers and trimers on large-scale substrates. Dynamic exponents are also studied by finite-size scaling of the spectrum gaps of evolution operators.
\end{abstract}

DOI: 10.1103/PhysRevE.88.052408

PACS number(s): 81.15.Aa, 02.50.-r, 05.10.Gg, 75.10.Jm

\section{INTRODUCTION}

The theory of surface growth processes has by now reached a mature status that allows one to describe statistically a wide variety of nonequilibrium phenomena in terms of universality classes of scaling regimes [1-3]. As is known, these latter depend strongly on the conservation laws of the underlying dynamics, bringing about important effects at long times. An interesting example of this, introduced in the context of restricted solid-on-solid dimer-growing interfaces [4,5], is the set of parity-conserving (PC) processes [2,3]. Here the seemingly microscopic variation of considering the dynamics of extended objects (i.e., involving more than one interface location) rather than that of monomers has, however, far reaching implications, giving rise to an anomalous growth of the global roughness or interface width. In one dimension, where nonequilibrium roughening transitions can also take place [4], this anomaly has been investigated in terms of even-visiting random walks [5]. In that representation the height levels of the interface are thought of as the visited sites of a one-dimensional (1D) Brownian path extended on a given time interval, here playing the role of the substrate length $L$. The constraint to cover each path location an even number of times (or, more generally, conserving this number modulo $k \geqslant 2$ ) introduces long-range temporal correlations [6], which causes the interface to roughen as $\sim L^{1 / 3}$ [5]. This is in marked contrast to the usual root mean square displacement of normal (diffusive) random walks characterizing the asymptotic $L^{1 / 2}$ width of a variety of interfaces grown out of monomers [1-3] and typical of both 1D Edwards-Wilkinson (EW) [7] and Kardar-Parisi-Zhang (KPZ) [8] universality classes. Also, roughening anomalies were reported in other growth models with similar global constraints including multiparticle correlations [9], self-flattening, and self-expanding surfaces [10].

In this work we examine further aspects of the PC processes referred to above, focusing attention on more detailed levels of description such as height difference correlation functions and width probability distributions. Interestingly and in line with a variety of studies of several growth models [11-15], it will turn out that in approaching the stationary regime there is a single length scale, namely, the usual average width, that characterizes these latter distributions in terms of a universal scaling function. Here we follow an alternative description of PC interfaces [16] using a simple extension of the well known 1D mapping between stochastic dynamics of binary lattice gases and body-centered solid-on-solid (BCSOS) growth processes $[1-3,17]$. There the differences of adjacent pairs of height variables $h_{n}$ are restricted to \pm 1 , while, as shown in Fig. 1, attachment and detachment of dimers (or $k$-mers in general) are viewed as exchanges of Ising spins $s_{n} \equiv$ $h_{n+1}-h_{n}$ on three $(2 k-1)$ consecutive bonds. Although the adsorbed particles do not diffuse explicitly in either $k$-mer or monomer form, they are allowed to rearrange throughout the interface by explicit dissociation of $k$-mers. This takes place under desorption attempts that may occur whether the $k$ targeted monomers were original adsorbing partners or not. In passing, it is worth mentioning that this is also a typical feature of catalytic surface reactions where the reconstitution of composite objects actually does matter $[2,3,18]$.

The simplicity of these rules is deceptive as they entail a number of conservation laws that grows exponentially with the substrate size. At the root of this rather unusual partitioning of the phase space is a useful construction, namely, the irreducible string, introduced by Barma and Dhar in closely related systems [19]. We shall exploit this nonlocal construct, defined later in Sec. II A, using a simple numerical algorithm that enables an approach to the stationary behavior without actually evolving the system. To that aim, we must content ourselves with analyzing just the situation of equal deposition and evaporation rates. The idea, to become clear in a moment, is to concatenate parts of steady configurations of initially flat but small interfaces such that the final assembly also bears the global constraint of an originally flat but much larger substrate. This approximation circumvents the problem of going through the slow subdiffusive dynamics characteristic of these processes $[2-5,16]$ (thus permitting a thorough sampling of width distributions in scaling regimes), which otherwise would be hard to examine by standard simulations. Nonetheless, to probe simple features of our assembled interfaces under the actual dynamics, we shall also make use of those simulations in large yet accessible scales. To complement this finite-size approach we also focus attention on dynamic exponents that, as is known [20], can be read off from the spectral gaps of evolution operators. Thus we shall 


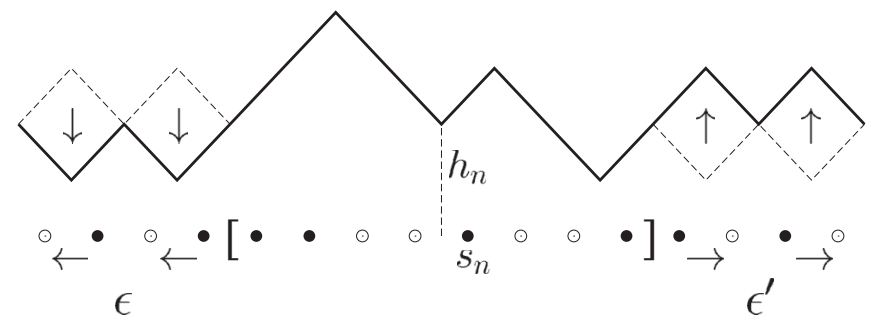

FIG. 1. Microscopic rules of a dimer-growing BCSOS interface and its equivalent 1D lattice gas. The dynamics of the former involves deposition (evaporation) of dimers with rate $\epsilon\left(\epsilon^{\prime}\right)$ at random locations having at least two consecutive height minima (maxima). The corresponding spin- $\frac{1}{2}\left(S_{n} \equiv h_{n+1}-h_{n}\right)$ or hard-core particle dynamics consists, respectively, of two simultaneous left (right) particle hoppings. The array between square brackets illustrates an irreducible string (see Sec. II A). Also, note that the identity of dimers is not necessarily maintained by subsequent evaporations.

diagonalize the latter exactly in reachable dimensions. In line with what was mentioned before, these exponents will end up being subdiffusive and in close agreement with those obtained by the usual dynamic scaling of the interface width [21].

The layout of this work is organized as follows. In Sec. II we recast the master equation of these processes in terms of a quantum spin Hamiltonian through which we not only obtain dynamic exponents but also span the entire phase space of small substrates. Following Ref. [19] to identify the conservation laws of this $k$-mer dynamics and utilizing the full configuration lists provided by the spanning, we then put forth our algorithm to sample rapidly the phase space of large interfaces. In Sec. III, at first we test this sampling method so as to retrieve known roughening exponents as well as steady-state correlations of smaller systems. Then we carry on with the evaluation of width distributions for which the onset of a universal scaling function is suggested. Surprisingly, the later exhibits a longer tail than that of normal random-walk-like interfaces [11]. Section IV contains a concluding discussion along with brief remarks on open issues and possible extensions of this work.

\section{DYNAMICS OF $\boldsymbol{k}$-MER INTERFACES}

The stochastic dynamics of discrete Markovian systems such as those referred to above amounts to a generic prescription of transition probability rates $R\left(S \rightarrow S^{\prime}\right) \geqslant 0$ between all possible configurations $S, S^{\prime}$ explored in time (here taken as being continuous). Therefore, the evolution of the probabilities $P(S, t)$ to observe the system in one of the latter is controlled by a gain-loss relation known as the master equation [22], namely,

$$
\partial_{t} P(S, t)=\sum_{S^{\prime} \neq S}\left[R\left(S^{\prime} \rightarrow S\right) P\left(S^{\prime}, t\right)-R\left(S \rightarrow S^{\prime}\right) P(S, t)\right] .
$$

Conveniently, this relation can also be rewritten in the form of a Schrödinger equation in imaginary time, that is, $\partial_{t}|P(t)\rangle=$ $-H|P(t)\rangle$, thus permitting one to derive the probability distribution $|P(t)\rangle \equiv \sum_{S} P(S, t)|S\rangle$ at subsequent moments from the action of $H$ on a given initial condition, i.e.,
$|P(t)\rangle=e^{-H t}|P(0)\rangle$. Here the Liouville or evolution operator $H$ embodying the dynamics is defined through its matrix elements

$$
\left\langle S^{\prime}|H| S\right\rangle=\left\{\begin{array}{lll}
-R\left(S \rightarrow S^{\prime}\right) & \text { for } & S \neq S^{\prime} \\
\sum_{S^{\prime} \neq S} R\left(S \rightarrow S^{\prime}\right) & \text { for } & S=S^{\prime},
\end{array}\right.
$$

which, due to conservation of probability, clearly constrain all $H$ columns to add up to zero. Thereby it can be shown [22] that the steady state corresponds to a unique $H$ eigenmode with eigenvalue $\lambda_{0}=0$, whereas the relaxation time of any observable is upper bounded by $1 / \operatorname{Re}\left(\lambda_{1}\right)>0$, with $\lambda_{1}$ being the first excitation level of the $H$ spectrum.

In our case, for what follows it is helpful to think of this evolution operator as being applied to a space of $\frac{1}{2}$-spinors. To do that we interpret the slope configurations $|S\rangle \equiv\left|S_{1}, \ldots, S_{L}\right\rangle$ of Fig. 1 as being already diagonal in the $z$ component, say, of Pauli matrices $\vec{\sigma}_{1}, \ldots, \vec{\sigma}_{L}$ assigned to each slope site. By construction it is then clear that up to a constant $h_{1}$ chosen as a reference level, the heights of the BCSOS interface are obtained as

$$
h_{n}=h_{1}+\sum_{j<n} S_{j}
$$

In particular note that under periodic boundary conditions (PBCs) the dynamics is consistent with a vanishing total magnetization $S^{z} \equiv \sum_{j} \sigma_{j}^{z}$, though, as we shall see below, many further additional conservation laws also emerge. Introducing now the right and left $k$-mer hopping operators $A^{+}, A^{-}$,

$$
A_{j}^{ \pm}=\prod_{i=1}^{k} \sigma_{j+2 i-1}^{ \pm} \sigma_{j+2 i-2}^{\mp},
$$

associated, respectively, with the detachment and attachment processes described in Fig. 1, and taking into account the algebra of the spin- $\frac{1}{2}$ raising and lowering operators $\sigma_{j}^{+}, \sigma_{j}^{-}$, we can readily write down the operational counterpart of Eq. (2), which here reduces to

$$
H=\sum_{j}\left(\epsilon^{\prime} A_{j}^{+}+\epsilon A_{j}^{-}\right)\left(A_{j}^{+}+A_{j}^{-}-1\right) .
$$

Such simplicity is only apparent as the commutation algebra of the hopping operators complicates the analytical treatment (except for monomers and $\epsilon=\epsilon^{\prime}$, where $H$ reduces to the isotropic Heisenberg ferromagnet). In the latter equation evidently each of the off-diagonal terms provides the appropriate transition elements of the dynamics, whereas the addition of its diagonal parts $B^{ \pm} \equiv \sum_{j} A_{j}^{ \pm} A_{j}^{\mp}$,

$$
\begin{aligned}
& B^{+}=\sum_{j} \prod_{i=1}^{k} \hat{n}_{j+2 i-2}\left(1-\hat{n}_{j+2 i-1}\right), \quad \hat{n}_{j} \equiv \sigma_{j}^{+} \sigma_{j}^{-}, \\
& B^{-}=\sum_{j} \prod_{i=1}^{k}\left(1-\hat{n}_{j+2 i-2}\right) \hat{n}_{j+2 i-1},
\end{aligned}
$$

accounts for the number of manners $N_{S}^{ \pm}=\left\langle S\left|B^{ \pm}\right| S\right\rangle$ in which a given spin configuration may access other ones by either right or left jumps, i.e., $\sum_{S^{\prime} \neq S} R\left(S \rightarrow S^{\prime}\right)=\epsilon^{\prime} N_{S}^{+}+\epsilon N_{S}^{-}$, thus complying with conservation of probability. In contrast, combining this with the microscopic reversibility of our model, 
here expressed simply as $R\left(S \rightarrow S^{\prime}\right)=\epsilon^{\prime}($ or $\epsilon) \Longleftrightarrow R\left(S^{\prime} \rightarrow\right.$ $S)=\epsilon\left(\right.$ or $\left.\epsilon^{\prime}\right)$, we then obtain

$$
\sum_{S^{\prime} \neq S}\left[R\left(S^{\prime} \rightarrow S\right)-R\left(S \rightarrow S^{\prime}\right)\right]=\left(\epsilon-\epsilon^{\prime}\right)\left(N_{S}^{+}-N_{S}^{-}\right),
$$

from which some brief remarks about the steady-state distribution now follow. First, note that the monomer case is special in that for PBCs (hereafter considered throughout) the latter identity always cancels out as for $k=1 \mathrm{Eq}$. (6) simplifies to $B^{+}=B^{-}=\frac{1}{4} \sum_{j}\left(1-\sigma_{j}^{z} \sigma_{j+1}^{z}\right)$. Therefore, comparing Eq. (7) with the right-hand side of the master equation, we thus see that the monomer steady state, either in equilibrium or not $\left(\epsilon \neq \epsilon^{\prime}\right)$, is consistent with a constant distribution [23]. More generally, however, except for the equilibrium situation, this feature does not hold for $k \geqslant 2$ because the diagonal $B^{+}, B^{-}$operators of Eq. (6) are now different, so in general $N_{S}^{+} \neq N_{S}^{-}$. Since the equiprobability issue is essential for the sampling algorithm that follows, this breakdown will restrict the numerical findings of Sec. III only to the case $\epsilon=\epsilon^{\prime}$, yet being nontrivial for $k \geqslant 2$, as we shall see below.

\section{A. Irreducible strings}

Turning to conservation laws and assuming that the lattice $\Lambda=\Lambda_{1}+\ldots+\Lambda_{2 k}$ is $2 k$ partite (in one dimension just meaning $L$ multiples of $2 k$ ), the first invariant set of quantities one can readily identify from the composite spin exchanges of Fig. 1 is that of the sublattice magnetization differences

$$
D_{n, m}^{z} \equiv \sum_{j \in \Lambda_{n}} \sigma_{j}^{z}-(-1)^{n+m} \sum_{j \in \Lambda_{m}} \sigma_{j}^{z},
$$

with $n, m=1, \ldots, 2 k$. From these $\left(\begin{array}{c}2 k \\ 2\end{array}\right)$ possible pairs only $2 k-1$ of them are independent, so the number of conservation laws would grow at most as $L^{2 k-1}$. However, as mentioned earlier, there is in fact a much subtler set of constants of motion, in turn growing exponentially with the lattice size.

To construct that set, here we briefly survey the ideas of Ref. [19] concerning the dynamics of the deposition and evaporation of trimers reconstructing on a line; for what follows it is convenient to adapt to larger objects of even length, e.g., $\bullet \bullet \bullet \rightleftharpoons \circ \circ \circ \circ$. Clearly, those processes are then isomorphic to ours via a simple particle-hole mapping, say, on even sublattices. Thus, in analogy to Ref. [19] we now define the irreducible string (IS) $I\left\{S_{1}, \ldots S_{L}\right\}$ of any spin configuration as the sequence obtained by deleting all groups of $2 k$ consecutive antiparallel spins appearing on chosen locations and then repeating recursively the procedure on the resulting shorter string until no further such groups remain. As an illustration, consider for simplicity the following examples of $k=2$ :

$$
\begin{aligned}
I\{\uparrow \downarrow \downarrow \uparrow \downarrow \uparrow \downarrow \uparrow\} & =I\{\uparrow \downarrow \downarrow \uparrow \downarrow \downarrow \uparrow \downarrow \uparrow\}=\{\uparrow \downarrow \downarrow \uparrow\}, \\
I\{\uparrow \downarrow \downarrow \uparrow \downarrow \uparrow \uparrow \downarrow\} & =\{\emptyset\} \text { (null string), } \\
I\{\uparrow \uparrow \downarrow \downarrow \uparrow \downarrow \downarrow \uparrow\} & =\uparrow \uparrow \downarrow \downarrow \uparrow \downarrow \downarrow \uparrow .
\end{aligned}
$$

In the first case this deletion, marked by boxes, is applied to a group of spins chosen starting from either the left or right. In the second instance the procedure is carried out recursively in two steps and no characters are left. In the

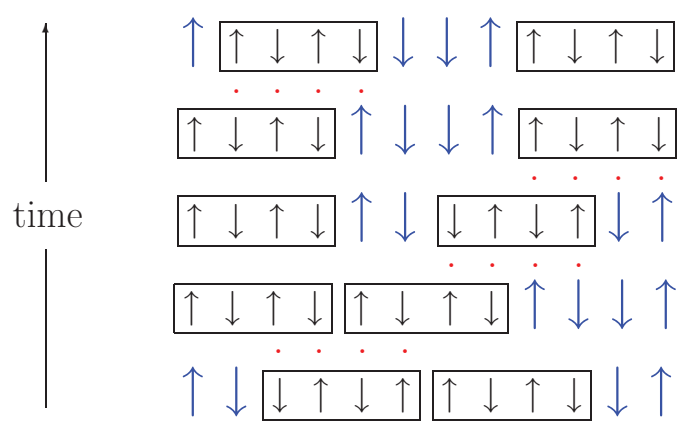

FIG. 2. (Color online) Schematic random walk of irreducible characters obtained by the deletion process described in the text, here drawn as boxes around reducible groups of spins. At each step the identification of the latter is made from left to right. Larger spins denote the irreducible characters whose ordering is left invariant by the dynamics. Dots signal the locations of updated spins.

third example the string considered is already jammed (same irreducible block of Fig. 1) and cannot evolve further. The invariance of the irreducible characters (if any) left by this process is in line with the idea that the successive action of the hopping operators of Eq. (4) on a given spin configuration just changes the position of those characters by multiples of $2 k$ lattice spacings. The separations between them are mediated by substrings of different lengths (proportional to $2 k$ ), though all of these are in turn reducible to null strings. Thus the interface dynamics may be thought of as a random walk of hard-core irreducible characters (they cannot cross each other), as depicted schematically in Fig. 2. The positions of these walkers at a given instant of course depend on the order in which the reduction rule is applied, but the key issue to bear in mind here is that the sequence of irreducible characters remains unaltered throughout. In addition, as noted in Ref. [19], two spin configurations $|S\rangle,\left|S^{\prime}\right\rangle$ are connected by the dynamics $\Longleftrightarrow I\{S\}=I\left\{S^{\prime}\right\}$. Thus the IS uniquely labels all subspaces left invariant by the $k$-mer kinetics, regardless of the order in which the reducible groups are removed. In contrast, it is clear that the number of combinations forming these irreducible sequences grows exponentially with the number of characters or string length $\mathcal{L} \leqslant L$. More specifically, a straightforward analysis of a recursion relation for this length $[19,24]$ shows that for large $\mathcal{L}$ and $k>1$ the number of invariant subspaces increases as fast as $x^{\mathcal{L}}$, where $x$ is the largest root of $x^{2 k}=2 x^{2 k-1}-1$.

On a more fundamental level, it would be interesting to identify the symmetries at the origin of these conservation laws. For instance, in the much simpler case of the sublattice differences of Eq. (8) the symmetries responsible for them just involve $\theta_{1}, \ldots, \theta_{2 k}$ rotation angles around the $z$ direction of each sublattice. Recalling that under those rotations $\sigma^{ \pm}$ transform as $e^{i \pm \theta} \sigma^{ \pm}$, evidently as long as $\sum_{n}(-1)^{n} \theta_{n}=0$ is held, the hopping operators of Eq. (4) will be left invariant and so will $H$ in Eq. (5). Thus, from the infinitesimal generators $\sum_{n}(-1)^{n} \theta_{n}\left(\sum_{j \in \Lambda_{n}} \sigma_{j}^{z}\right)$ of these $2 k-1$ independent rotations one is finally led to the conservation laws of Eq. (8), already obtained on more intuitive grounds. However, in the case of the IS and the exponential proliferation of constants of motion 
it entails, the analysis appears to be much more involved. Due to the highly convoluted form in which the IS is obtained, unlike Eq. (8) it is not clear how to construct its operational counterpart (possibly nonlocal) or identify the corresponding symmetries in the evolution operator. Despite that formal insufficiency, the invariance of the IS provides an alternative computational tool to approach the equilibrium regime, which we now implement.

\section{B. Assembling null string states}

Notice that whenever $\rho=\mathcal{L} / L$ is kept finite in the thermodynamic limit, the interface cannot roughen at large times [16]. This is because for $\epsilon=\epsilon^{\prime}$ the distances $\lambda$ between irreducible characters (or random walkers) are distributed as $\simeq \rho e^{-\rho \lambda}[25]$. Thus mean square height fluctuations along those distances (or reducible substrings, all with $S^{z}=0$ ) remain bounded as $1 / \rho^{3}$. Thus, hereafter we will focus on the null string subspace only. Besides, it is the most natural to consider in the context of growing interfaces, as it stems from initially flat conditions (plain antiferrous states).

Although in equilibrium all configurations are equally weighted, the expectation value of most observables are not analytically simple to obtain because the ensemble of averaged states must be consistent not just with $S^{z}=0$, but also with a vanishing IS. This introduces spatial correlations (absent in the monomer case, where $\left|\sum_{j} S_{j}\right|$ always coincides with $\mathcal{L}$ ), which develop slowly in the course of growth simulations. At long times, however, such a process ultimately amounts to producing a uniform distribution of null string states. Here we put forth an approximation of such a distribution in large-scale substrates based on the construction of small ones. The idea is to assemble pieces of small substrates in such a way that by applying the above deletion rules the whole set is reducible to the null string.

There are several forms to attempt this, but consider, for instance, a set of $2^{N}$ spin configurations (sketched as the initial blocks of Fig. 3) with a common length $L_{0} \propto 2 k$ and drawn randomly from a list of null string blocks previously prepared. The latter, in turn, can be constructed from the repeated action of the hopping operators (4) on, say, initial antiferrous states, until exhausting the full space (typically growing exponentially with $L_{0}$ [19]). At the first step, half of the drawn blocks are divided in two parts at random locations, while as depicted in Fig. 3 each of the remaining blocks is settled between the split pairs. Thus one is left with $2^{N-1}$ blocks of length $2 L_{0}$, all of which are evidently reducible to the null string [see, e.g., Eq. (9)]. Next, the process is recursed, eventually by splitting further parts of the original blocks, until a single block of length $2^{N} L_{0}$ is obtained. The algorithm thus generates an ensemble of substrates that are fully reducible by successive reductions around a central $L_{0}$ block, always left unsplit. Though uniform, this assembled distribution (AD) cannot be entirely representative of the much larger substrate space; at most it just can be approximative. Nevertheless, as will be tested in Sec. III, it does reproduce known features of the scaling regimes in which one is ultimately interested while enabling one to examine there width distributions otherwise difficult to reach. Let us finally comment that had

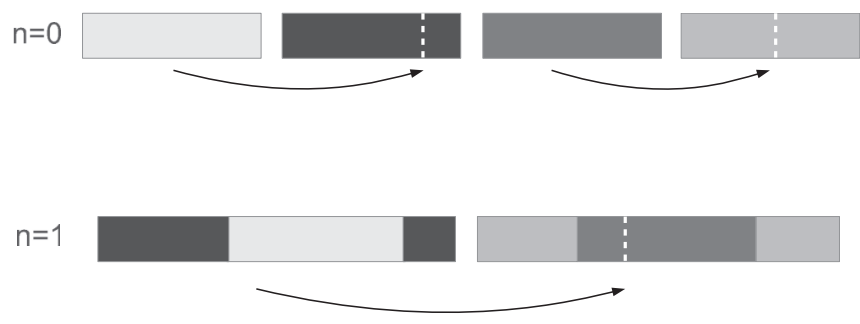

$\mathrm{n}=2$

FIG. 3. Schematic representation of the sampling algorithm after two iterations. The initial blocks stand for null string configurations drawn randomly from all possible ones constructed for an accessible lattice size $L_{0}$. Dashed lines denote random locations where an entire block is interposed. At each step the block size is doubled while complying with the null string constraint. Eventually, parts of the original blocks can also be further split by random intercalations.

a simple concatenation of blocks been carried out it would have bounded all heights as $\left|h_{j}\right| \leqslant L_{0} / 2$, whereas to the other extreme, the use of more reduction centers would not bring about a better approximation to the equilibrium regime.

\section{NUMERICAL RESULTS}

Before applying this algorithm to width distributions, we first test it against typical scaling aspects of growing interfaces. In studying the latter one usually considers the mean square fluctuations of the average height $\bar{h}(t) \equiv \frac{1}{L} \sum_{j} h_{j}(t)$, which provides a measure of the global interface width at a given instant, that is,

$$
W^{2}(L, t)=\frac{1}{L} \sum_{j}\left\langle\left[h_{j}(t)-\bar{h}(t)\right]^{2}\right\rangle .
$$

Here the angular brackets denote an ensemble average over all possible evolutions of heights, in our case compatible with the null string imposed by flat initial conditions. Based on a wide range of theoretical and numerical studies, it can be argued that $W^{2}$ should scale as $[1-3,21]$

$$
W^{2}(L, t) \simeq L^{2 \zeta} f\left(t / L^{z}\right),
$$

with a universal scaling function behaving as $f(x) \propto x^{2 \zeta / z}$ for $x \ll 1$, while approaching a constant for $x \gg 1$. Hence, for $t \ll L^{z}$ the width must grow as $t^{\zeta / z}$ [26], until saturating as $L^{\zeta}$ for times comparable to or larger than the relaxation time $\tau$. In the above hypothesis the Hurst or roughening exponent $\zeta$ measures the stationary dependence of the interface width on the typical substrate size, while the fundamental scaling between length and time is given by the dynamic exponent $z$.

\section{A. Scaling exponents}

When it comes to the latter it is helpful to also consider the spectral gap $\operatorname{Re}\left(\lambda_{1}\right)=1 / \tau$ of the evolution operator constructed in Sec. II so as to obtain a separate evaluation (independent of $\zeta$ ), which we now briefly touch upon. 


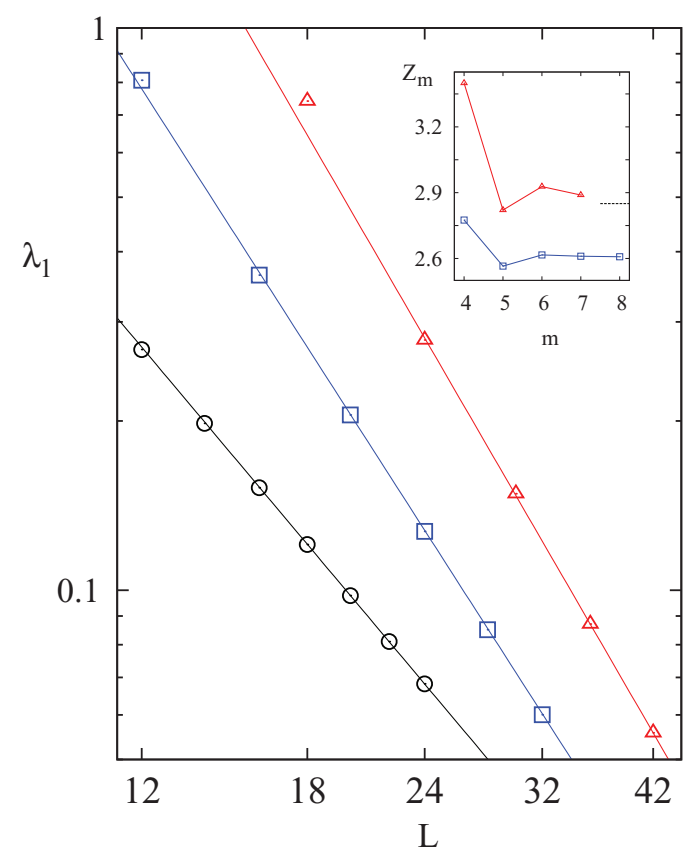

FIG. 4. (Color online) Finite-size decay of spectral gaps of evolution operators [restricted to $\epsilon=\epsilon^{\prime}$ in Eq. (5)] for dimers (squares), trimers (triangles), and monomers (circles, just for comparison). From top to bottom solid lines are fitted with slopes corresponding to dynamic exponents $z(k) \simeq 2.89,2.61$, and 2 , obtained in turn from the highest available approximants of Eq. (12). These are exhibited in the inset for $k=2$ and 3 using several lattice sizes $(L=2 m k$ ). The horizontal line indicates the value of $z(3) \simeq 2.85$ arising from Fig. 5(b).

Assuming as usual the emergence of a finite-size scaling regime in which $\tau \propto L^{z}$ [20], we analyze those exponents for $\epsilon=\epsilon^{\prime}$ and small substrates using a Lanczos diagonalization [27] of Eq. (5) within null string subspaces in turn spanned via the hopping operators (4) as explained in Sec. II B. The results so obtained are displayed in Fig. 4, where it is shown that already modest lengths are able to yield clear finite-size trends that evidence nonuniversal and subdiffusive slopes for dimers and trimers, i.e., $z \simeq 2.61$ and 2.89 , respectively. In that regard, a convergence estimation of these values can be made by defining the sequence of dynamic exponents or approximants

$$
z_{m}=\frac{\ln \left[\lambda_{1}\left(L_{m}\right) / \lambda_{1}\left(L_{m-1}\right)\right]}{\ln [(m-1) / m]},
$$

with $L_{m} \equiv 2 \mathrm{~km}$. As shown in the inset of Fig. 4, the relative differences between our highest approximants are about $0.1 \%$ for $k=2$ and $1.3 \%$ for $k=3$, which in any case are far apart from the diffusive slope $(z \simeq 1.99)$ of the monomer case, only shown for comparison. It would be desirable to improve the convergence of the trimers $z$, but the next approximant $(L=48)$ requires one to consider spaces of more than $2.6 \times 10^{7}$ null string states, which goes beyond our computing facilities. Nevertheless, we can compare these exponents with those arising from the standard scaling hypothesis (11) while, more importantly, testing the validity of the type of scheme previously proposed.
To this aim, we compare the evolutions of flat substrates with those resulting from the AD of Sec. II B. This we do in Fig. 5, where the scaled widths of these two different preparations are displayed for not too large sizes, so as to reach about $10^{4}$ samples in the final saturation regime (recall that $z$ is subdiffusive). The sets exhibit different scaling functions according to the substrate preparations, but in both cases the data collapse was attained upon setting a common roughening exponent $\zeta \simeq 0.3(1)$ (for either $k=2$ or 3 ), along with common dynamic ones $z \simeq 2.60(8)$ and 2.85(3) for dimers and trimers, respectively. Interestingly, the two latter values happen to follow closely those of the approximants referred to above (inset of Fig. 4), while, in contrast, the early time widths arising from the AD already scale around a significant fraction of their asymptotic values, i.e., $\sim 75 \%$ for dimers and $\sim 84 \%$ for trimers. This trend still improves when assembling larger $L_{0}$ blocks, namely, the scaled widths approach larger fractions of the saturation values observed in Fig. 5 while keeping a common roughening exponent $\zeta \simeq 0.29(3)$ pretty close to the value obtained above. In Fig. 6 this is corroborated for a variety of substrate sizes $2^{n} L_{0}(n=2, \ldots, 12)$ assembled with several $L_{0}$ blocks, otherwise unreachable by standard simulations. In that sense note that the algorithm of Sec. II B is not severely limited by the number of recursions $n$, but rather by the large list of null string states increasing exponentially with $L_{0}$. Once the latter are evaluated, the algorithm permits one to rapidly average over about $10^{6}$ samples of rather large lattices.

As we move toward more detailed levels of description, we now consider the height difference correlation functions for which a similar scaling hypothesis is also expected to hold at distances $|r| \ll L$, that is [1-3,21],

$$
D_{L}^{2}(r, t)=\frac{1}{L} \sum_{j}\left\langle\left[h_{j+r}(t)-h_{j}(t)\right]^{2}\right\rangle \simeq|r|^{2 \zeta} g\left(t /|r|^{z}\right) .
$$

As before, the angular brackets are taken as in Eq. (10) and the scaling function $g(x)$ behaves analogously to that of Eq. (11). From this one infers that a time $t \sim|r|^{z}$ is required to fully develop the interface roughness across a given distance $|r|$. Thus, to check whether our AD can already be associated with late stages of growth, we measure these height correlations using over three distance decades for several $L_{0}$ substrates. The results are shown in the upper insets of Fig. 6, where it is shown that in all cases the previous roughening exponent $\zeta \simeq 0.29(3)$ is recovered. For display purposes we rescaled these data with the same $s_{0}$ amplitudes $\left(1.3 \lesssim s_{0} \lesssim 1.5\right)$ used in the main panels, which is no coincidence, as it would be expected on the basis of the identity $\lim _{r \rightarrow \infty} \lim _{L \rightarrow \infty} D_{L}^{2}(r)=\lim _{L \rightarrow \infty} 2 W_{L}^{2}$.

Before continuing we pause to comment on the differences appearing between the exponents of the dimer dynamics and those in the even-visiting random walks (EVRW) analyzed in Ref. [5]. Our $\zeta$ value should not be regarded as a mere numerical deviation from the 1/3 exponent conjectured in [5]. At the origin of this departure is the exponential proliferation of irreducible strings appearing in the dimer dynamics which ultimately impose tighther restrictions than those already occurring in EVRW. Although both dynamics share the topological constraint caused by the mod 2 conservation of the number of particles at every height level, note that the BCSOS version of EVRW [5] mixes up all the many-sector decomposition discussed in Sec. II A. For instance, besides the dimer 

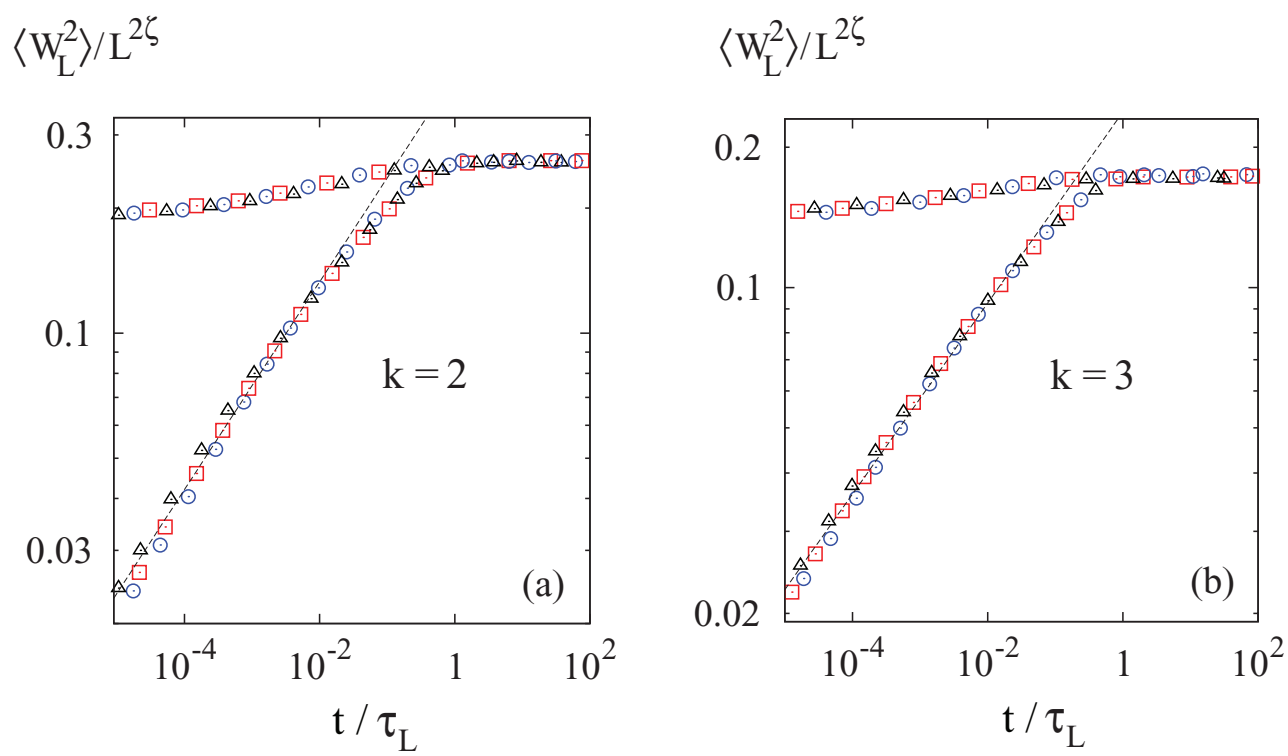

FIG. 5. (Color online) Dynamic scaling of interface widths using substrates of sizes $L=2^{n} L_{0}$ for (a) dimers $\left(L_{0}=32\right)$ and (b) trimers $\left(L_{0}=36\right.$ ). Circles, squares, and triangles stand for $n=4,5$, and 6 , respectively. The uppermost set of data was initially prepared from the distributions of Sec. II B, while the lowermost set was started by the usual flat conditions. The scaling of data was obtained setting $\zeta \simeq 0.3$, and $\tau_{L}(k)=A_{k} L^{z(k)}$ with $z(2) \simeq 2.61, z(3) \simeq 2.85$, and amplitudes $A_{2}, A_{3}$ estimated from the reciprocal ones of Fig. 4. The slopes of the straight lines are fitted with values $2 \zeta / z$.

dynamics Ref. [9] also considers the full restoration of ergodicity by deposition-evaporation of two particles at two randomly chosen columns with equal heights. These need not to be contiguous (as in the dimer dynamics), nor necessarily share the same terrace. That introduces a genuine one-to-one correspondence with the ensemble generated by the EVRW dynamics, so the estimate of $\zeta \sim 0.33$ found there in such conditions is then in line with the theory of Ref. [5]. Similarly, that latter work also investigates the effect of adding monomer diffusion within terraces. That produces another estimation which yields $\zeta \sim 0.31$. But further to that difference, note that the explicit addition of monomer diffusion partially relaxes the broken

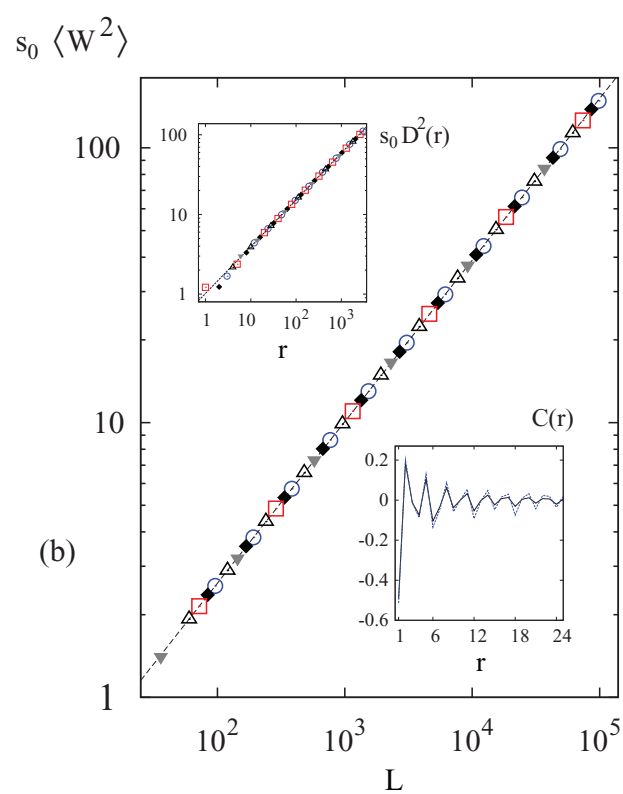

FIG. 6. (Color online) Finite-size growth of interface width for (a) dimers and (b) trimers recursing the algorithm of Fig. 3. In (a) the assembling uses blocks of $L_{0}=12,20,24,28$, and 36 sites (closed downward and open upward triangles, rhomboids, squares, and circles, respectively), while in (b) those symbols stand in turn for blocks of $L_{0}=18,30,36,42$, and 48 . The upper insets exhibit the height difference correlation functions of Eq. (13) using 12 recursions. Each set of $L_{0}$ data was normalized by an overall scale factor $s_{0}$, chosen in turn so as to fit the saturation amplitude obtained in Fig. 5. In all cases the scaling $W^{2} \propto L^{2 \zeta}$ and $D^{2}(r) \propto r^{2 \zeta}$ appears with a common roughening exponent $\zeta \simeq 0.29$. The lower insets display comparisons of assembled slope correlations of Eq. (14) (solid lines), with actual steady ones in small lattices (dashed lines). 
ergodicity of the original dimer dynamics. Hence, the above deviations of $\zeta$ from $1 / 3$ should not be ascribed merely to statistical errors but mainly to the change of conservation laws.

In fact, when restoring full ergodicity Ref. [9] yields a KPZ type exponent $z \sim 1.5$ which is far apart from the subdiffusive $z \sim 2.6$ obtained in the original dimer dynamics of both Refs. [5] and [9] as well as in this section.

Turning to smaller scales and to further probe the AD, we finally compare the exact slope or spin correlations

$$
C(r)=\frac{1}{L} \sum_{j}\left\langle S_{j+r} S_{j}\right\rangle,
$$

evaluated in the uniform distributions of our largest available blocks (36 heights for dimers and 48 for trimers), with those estimated in substrates assembled with smaller $L_{0}$. This is illustrated in the lower panels of Fig. 6, where it is corroborated that in both cases these functions closely approximate each other. As mentioned earlier, we thus see that even though the averaged distributions are uniform, the null string constraint enforces nontrivial correlations that otherwise would not appear by the sole restriction of $S^{z}=0$ (as in the case of monomers, where all even correlators $\left\langle S_{j_{1}}, \ldots, S_{j_{2 n}}\right\rangle$ vanish identically as $L^{-n}$ ). Noting that either Eq. (10) or (13) can also be expressed in terms of $C(r)$, it follows that for $k \geqslant 2$ these pair correlations are ultimately responsible for the anomalous roughening of PC interfaces, as opposed to the case $k=1$ where these pairs (proportional to $L^{-1}$ ) have no effect.

\section{B. Width distribution}

It is reassuring that both roughening exponents and correlations of Fig. 6, in conjunction with the near-saturated scaling regimes of Fig. 5, suggest strongly that the construction of $\mathrm{Sec}$. II B is sampling close-to-equilibrium states. Thus we carry on and further exploit that construction to compute the width distributions of PC interfaces.

Since the dynamic scaling hypothesis (11) involves in fact an integral over all interface modes, it may well occur that corrections to scaling are needed. In our case, this is particularly noticeable at the early stages of evolution where the data collapse in the lower sets of Figs. 5(a) and 5(b) is not so evident. In that sense, a variety of theoretical and numerical studies [11-15] have suggested an alternative characterization of interfaces in terms of the full probability distribution $P\left(w^{2}\right)$ of its particular random width realizations $w^{2}$. So long as their average $\left\langle W^{2}\right\rangle$ diverges in the thermodynamic limit, i.e., $\zeta>0$, the relevance of such a distribution relies on that for large substrates it scales as [11-15]

$$
P_{L}\left(w^{2}\right) \simeq \frac{1}{\left\langle W_{L}^{2}\right\rangle} \Phi\left(\frac{w^{2}}{\left\langle W_{L}^{2}\right\rangle}\right),
$$

where the scaling function $\Phi(x)$ is a universal characteristic of the interface fluctuations. Like $\zeta$, however, at large times this function cannot point out dynamic aspects of universality classes. For example, since in one dimension the steady states grown out of $k=1$ are equiprobable and uncorrelated (recall Sec. II), $\Phi(x)$ is the same for both EW $\left(z=2, \zeta=\frac{1}{2}\right)$ and $\mathrm{KPZ}\left(z=\frac{3}{2}, \zeta=\frac{1}{2}\right)$ universality classes [11]. However, in view of the role of null strings for $k \geqslant 2$,

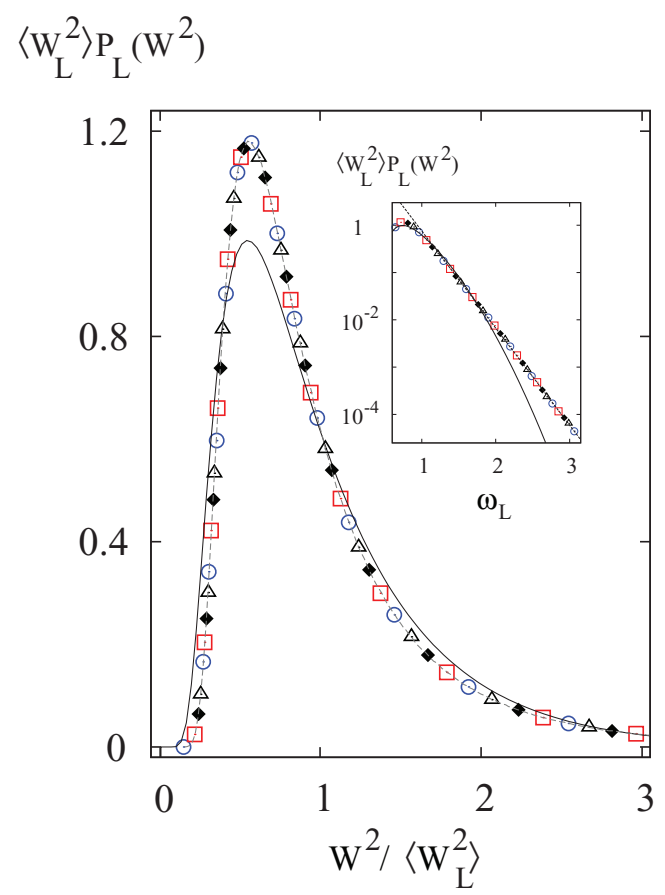

FIG. 7. (Color online) Scaling of width distributions close to equilibrium regimes of dimer and trimer interfaces for various lattice sizes $2^{n} L_{0}$. Squares and triangles stand for strings of $k=2$ assembled with $L_{0}=36$ and 24 using $n=10$ and 8 , respectively. In turn, circles and rhomboids denote $k=3$ strings stemming from blocks of $L_{0}=48$ and 30, also recursed 10 and 8 times in each case. For comparison, the solid line refers to the $k=1$ distribution of Ref. [11], whereas the dashed one is just a guide to the eye. The inset evidences a longer tail for $k \geqslant 2$, clearly decaying exponentially with $\omega_{L} \equiv W / \sqrt{\left\langle W_{L}^{2}\right\rangle}$.

$\Phi(x)$ should be able to distinguish clearly the growth of PC interfaces from that of monomer ones.

To that aim, note that the basic problem of sampling stationary $w^{2}$ under very slow relaxation $(z>2)$ inevitably arising in standard simulations is to a large extent bypassed by the close-to-equilibrium distributions of Sec. II B. In turn, these also enable one to sample sufficiently large lattices as otherwise there would be a rather small number of possible $w^{2}$ values coarse graining the histogram of $P_{L}\left(w^{2}\right)$. Thus, using substrates in the range of 6.000-50.000 heights, in Fig. 7 we display the scaled distributions obtained after dividing the data into $\simeq 10^{3}$ intervals (not all shown) and averaging over about $5 \times 10^{7}$ samples. This rather extended sampling, facilitated through the algorithm of Sec. II B, was necessary to capture the statistics of the rare events on the tail of the distribution given in the figure inset. As expected, in all cases the data collapse into a single scaling function easily discernible from the exact monomer result [11]. (The collapse is better demonstrated within the scales of the inset where probabilities are much smaller.) Furthermore, $\Phi(x)$ appears to be the same for dimers and trimers, each stemming from dissimilar types of assembled null strings, thus suggesting a universal function for equilibrium PC interfaces. In contrast to the scaling hypothesis (11), here note that there are no parameters to collapse the $\Phi$ and no scaling properties of $\left\langle W_{L}^{2}\right\rangle$ are used or assumed. The only approximation is the finite size of the systems 
investigated. In that regard, other large substrates assembled with different $L_{0}$ and $n$ (not displayed to avoid overcrowding) yielded the same, numerically indistinguishable, functions.

Just as the monomer case, we see that the length scale $L^{\zeta}$ not only characterizes the macroscopic level of the interface roughness [Eq. (11) and Fig. 6], but also emerges as the natural length of the whole width distribution. Since $\zeta<1 / 2$, one would intuitively presume that height fluctuations in PC interfaces are smaller than those in monomer ones. Thus, on approaching the above length scale one would expect $\Phi(x)$ to become more peaked and narrower than the monomer $\Phi$, something that in fact occurs to some extent. However, on the same basis one would also expect the PC distribution to decay faster for large width realizations. Surprisingly, however, it turns out to be the other way around. This is illustrated in the inset of Fig. 7, where the semilogarithmic plot strongly suggests an exponential decay in the scaled variable $W / \sqrt{\left\langle W^{2}\right\rangle}$ rather than its square, as occurs in the exact solution of $k=1$ [11]. More specifically, the tails of these two distributions behave as

$$
\Phi(x>1.5) \simeq\left\{\begin{array}{lll}
\frac{\pi^{2}}{3} \exp \left(-\frac{\pi^{2}}{6} x\right) & \text { for } & k=1 \\
a \exp (-b \sqrt{x}) & \text { for } & k=2,3,
\end{array}\right.
$$

with fitting parameters $a \simeq 85 \pm 2$ and $b \simeq 4.7 \pm 0.1$. Hence we conclude that even though the average roughness of finite PC interfaces is significantly smaller $(\zeta \simeq 0.29)$, their fluctuations can eventually explore larger widths.

\section{SUMMARY AND DISCUSSION}

We have presented an alternative approach to 1D parity-conserving interfaces close to their equilibrium regimes $\left(\epsilon=\epsilon^{\prime}\right)$. The notion of irreducible string [19], which partitions the dynamics into many disjoint sectors of the configuration space, played an instrumental role in the implementation of the assembling algorithm put forth in Sec. II B (Fig. 3). The latter was shown to provide a fair sampling of the almost saturated state, in turn exhibiting a scaling regime (Fig. 5) controlled by the very same dynamic and roughening exponents obtained through the dynamic scaling hypothesis (11) [21]. Without explicitly evolving the system in time, the assembled distribution also reproduced the latter exponent at both the macroscale of the average interface width (main panels of Fig. 6) and the microlevel of the height difference correlations of Eq. (13) (upper insets). The value of $\zeta \simeq 0.29(3)$ so obtained is also in excellent agreement with those resulting from simulations in previous studies [16] and with restricted solid-on-solid versions of these interfaces [4,5].

As for dynamic exponents, we diagonalized the evolution operator (5) within the accessible null string spaces already stored in assembling the above distributions so as to analyze the size dependence of its spectral gap (Fig. 4). This provided an estimation of dynamic exponents via a sequence of finitesize approximants (inset of Fig. 4) nearing subdiffusive but nonuniversal values, i.e., $z \simeq 2.61$ and 2.89 for dimers and trimers respectively, both in reasonable agreement with the exponents resulting from the scaling hypothesis (11).

Apart from avoiding the slow subdiffusive dynamics, perhaps the most interesting aspect of our assembling approach is that it also allows for a rich statistical analysis of the full width distribution of large scales. All assembled sizes yielded a single universal scaling function for both dimers and trimers (Fig. 7), in turn quite distinct from that of normal random-walk or monomer interfaces [11]. This contributes to the list of already known scaling functions [11-15] that concurrently with roughening exponents may be used to identify static universality classes of growth processes. The only length scale spontaneously emerging in those functions is the average interface width proportional to $L^{\zeta}$, which in the PC class is conspicuously smaller than in other classes. Curiously, however, height fluctuations in the former turn out to build up in such a way that tails of width distributions decay much slower than those in monomer interfaces [stretched exponential of Eq. (16) and inset of Fig. 7].

Returning to irreducible strings, note that in equilibrium the only one in which fluctuations diverge even in the thermodynamic limit is just the null string. All other ones containing a finite density $\rho=\mathcal{L} / L$ of irreducible characters might be considered as noncritical strings. In that regard, Fig. 2 is helpful to understand the latter as random sequences of null substrings of length $\lambda$ (distributed as $\rho e^{-\rho \lambda}[25]$ ), through which mean square height fluctuations cannot but remain bounded as $\sim \int_{0}^{\infty} \lambda^{2} \rho e^{-\rho \lambda} d \lambda=2 / \rho^{3}$. However, note that as soon as $\epsilon \neq \epsilon^{\prime}$, stationary probabilities immediately become nonuniform [recall discussion below Eq. (7)] and this simple picture no longer holds. Notwithstanding that the nonequilibrium dynamics is still partitioned by the same strings, it remains to figure out whether the current assembling approach, for either null or finite strings, could be extended to incorporate those nonuniform measures. Because of the latter, nonequilibrium width distributions no longer need to be related to the scaling function obtained here. Finally, in $d>1$, where there is no analog of irreducible string, all these issues, either in equilibrium or not, remain open.

\section{ACKNOWLEDGMENTS}

It is a pleasure to thank F. A. Schaposnik for helpful discussions. The authors acknowledge support from CONICET and ANPCyT, Argentina, under Grants No. PIP 1691 and No. PICT 1426.
[1] J. Krug, Adv. Phys. 46, 139 (1997); T. Halpin-Healy and Y.-C. Zhang, Phys. Rep. 254, 215 (1995); A.-L. Barbási and H. E. Stanley, Fractal Concepts in Surface Growth (Cambridge University Press, Cambridge, 1995); P. Meakin, Phys. Rep. 235, 189 (1993).

[2] G. Ódor, Universality in Nonequilibrium Lattice Systems. Theoretical Foundations (World Scientific, Singapore, 2008), Chap. 7; Rev. Mod. Phys. 76, 663 (2004).
[3] M. Henkel, H. Hinrichsen, and S. Lübeck, Non-Equilibrium Phase Transitions, Vol. 1 (Springer, Dordrecht, 2008), Chaps. 3-5.

[4] H. Hinrichsen and G. Ódor, Phys. Rev. Lett. 82, 1205 (1999); Phys. Rev. E 60, 3842 (1999).

[5] J. D. Noh, H. Park, and M. den Nijs, Phys. Rev. Lett. 84, 3891 (2000); J. D. Noh, H. Park, D. Kim, and M. den Nijs, Phys. Rev. E 64, 046131 (2001). 
[6] J.-P. Bouchaud and A. Georges, Phys. Rep. 195, 127 (1990); M. N. Barber and B. W. Ninham, Random and Restricted Walks, Theory and Applications (Gordon and Breach, New York, 1970), Chaps. 6 and 7.

[7] S. F. Edwards and D. R. Wilkinson, Proc. R. Soc. London Ser. A 381, 17 (1982).

[8] M. Kardar, G. Parisi, and Y.-C. Zhang, Phys. Rev. Lett. 56, 889 (1986).

[9] Y. Kim, T. S. Kim, and H. Park, Phys. Rev. E 66, 046123 (2002).

[10] Y. Kim, S. Y. Yoon, and H. Park, Phys. Rev. E 66, 040602(R) (2002); Y. Kim and S. Y. Yoon, ibid. 69, 027101 (2004).

[11] G. Foltin, K. Oerding, Z. Rácz, R. L. Workman, and R. K. P. Zia, Phys. Rev. E 50, R639 (1994).

[12] M. Plischke, Z. Rácz, and R. K. P. Zia, Phys. Rev. E 50, 3589 (1994); Z. Rácz and M. Plischke, ibid. 50, 3530 (1994).

[13] T. Antal and Z. Rácz, Phys. Rev. E 54, 2256 (1996); T. Antal, M. Droz, G. Györgyi, and Z. Rácz, Phys. Rev. Lett. 87, 240601 (2001); Phys. Rev. E 65, 046140 (2002).

[14] E. Marinari, A. Pagnani, G. Parisi, and Z. Rácz, Phys. Rev. E 65, 026136 (2002).

[15] P. Le Doussal and K. J. Wiese, Phys. Rev. E 68, 046118 (2003); A. Rosso, W. Krauth, P. Le Doussal, J. Vannimenus, and K. J. Wiese, ibid. 68, 036128 (2003); F. D. A. Aarão Reis, ibid. 72, 032601 (2005).

[16] M. D. Grynberg, J. Stat. Phys. 103, 395 (2001); Phys. Rev. E 76, 031605 (2007).

[17] M. Plischke, Z. Rácz, and D. Liu, Phys. Rev. B 35, 3485 (1987). For a recent two-dimensional realization of this mapping, see G. Ódor, B. Liedke, and K.-H. Heinig, Phys. Rev. E 81, 051114 (2010).
[18] J. Marro and R. Dickman, Nonequilibirum Phase Transitions in Lattice Models (Cambridge University Press, Cambridge, 1999), Chap. 5.

[19] M. Barma and D. Dhar, Phys. Rev. Lett. 73, 2135 (1994); D. Dhar and M. Barma, Pramana-J. Phys. 41, L193 (1993); M. Barma, ibid. 49, 155 (1997); in Nonequilibrium Statistical Mechanics in One Dimension, edited by V. Privman (Cambridge University Press, Cambridge, 1997).

[20] P. C. Hohenberg and B. I. Halperin, Rev. Mod. Phys. 49, 435 (1977).

[21] F. Family and T. Vicsek, J. Phys. A 18, L75 (1985); J. Kertész and T. Vicsek, in Fractals in Science, edited by A. Bunde and S. Havlin (Springer, Berlin, 1994).

[22] N. G. van Kampen, Stochastic Processes in Physics and Chemistry, 3rd ed. (North-Holland, Amsterdam, 2007), Chap. 5.

[23] B. Derrida, Phys. Rep. 301, 65 (1998), in particular Sec. 2.

[24] R. B. Stinchcombe, M. D. Grynberg, and M. Barma, Phys. Rev. E 47, 4018 (1993), in particular Appendix C.

[25] W. Feller, An Introduction to Probability Theory and its Applications, 2nd ed., Vol. 2 (Wiley, New York, 1970), Chap. 1.

[26] However, for the 1D PC interfaces being considered, the specific form of this scaling hypothesis holds only under detailed balance conditions. Otherwise, the width either exhibits a logarithmic growth or is kept bounded; see Refs. [4,5,16].

[27] See for example, G. H. Golub and C. F. van Loan, Matrix Computations, 3rd ed. (Johns Hopkins University Press, Baltimore, 1996), Chap. 9; Y. Saad, Numerical Methods for Large Eigenvalue Problems, 2nd ed. (SIAM, Philadelphia, 2011), Chap. 6. 